\title{
Reversibly growing crosslinked polymers with programmable sizes and properties
}

Joanna Aizenberg ( $\nabla$ jaiz@seas.harvard.edu )

Harvard University https://orcid.org/0000-0002-2343-8705

Jiaxi Cui

University of Electronic Science and Technology of China

Xiaozhuang Zhou

INM-Leibniz Institute for New Materials

Yijun Zheng

INM-Leibniz Institut for New Materials

Haohui Zhang

Georgia Institute of Technology

Li Yang

University of Electronic Science and Technology of China

Yubo Cui

University of Electronic Science and Technology of China

\section{Baiju Krishnan}

INM-Leibniz Institute for New Materials

\section{Shihua Dong}

University of Electronic Science and Technology of China

\section{Michael Aizenberg}

Harvard University

\section{An Zeng}

Shanghai Institute of Biochemistry and Cell Biology, Chinese Academy of Sciences

\section{Yuhang Hu}

Georgia Institute of Technology

\section{Physical Sciences - Article}

Keywords: polymers, Planaria, equilibration

Posted Date: July 16th, 2021

DOI: https://doi.org/10.21203/rs.3.rs-692617/v1 
License: (c) (i) This work is licensed under a Creative Commons Attribution 4.0 International License. Read Full License 


\section{Abstract}

Planaria can integrate external nutrients to grow or consume their bodies to degrow for adapting to environmental changes. In contrast, synthetic organic materials, especially those with crosslinked structures, are normally static and cannot post-vary their sizes and properties without compromising material mechanical performances for sustainable use. Inspired by Planaria, we here report a growingdegrowing strategy of enabling thermosetting materials to either absorb or release components for continuously changing their sizes, shapes, compositions, and a set of properties simultaneously. The strategy is based on the monomer-polymer equilibrium of networks in which supplying or removing small polymerizable components would drive the networks toward expansion or contraction. Using acidcatalyzed equilibration of siloxane as an example, we demonstrate that the size and mechanical properties of the resulting silicone materials can be significantly or finely tuned in both directions of growth and decomposition. The equilibration can be turned off to yield stable products or reactivated again. During the degrowing-growing circle, material structures are selectively varied either uniformly or heterogeneously, by the availability of fillers. Our strategy endows the materials with many appealing capabilities including environment adaptivity, self-healing, and switchability of surface morphologies, shapes, and optical properties. Since monomer-polymer equilibration exists in many polymers, we envision the expansion of the presented strategy to various systems for many applications.

\section{Main}

Planaria can constantly grow and degrow to meet environmental challenges, due to the self-organization of their living bodies, which are always in a food-dependent balance between absorbing nutrients to proliferate and consuming themselves to survive (Figure $1 \mathrm{~A})^{1}$. Such an open, equilibrium mechanism allows Planaria to vary their sizes and components in extremely broad ranges, and even to regenerate the whole body from a piece of them with incredible memory effect ${ }^{2}$. By contrast, synthetic soft materials are normally designed with specific properties and fabricated into given sizes. They are inert and cannot adapt to changing situations after they have been processed ${ }^{5-8,11}$. When external geometric requirements that the product should satisfy change, or different properties or performance of the material are needed, new products with updated sizes and properties are typically designed and manufactured, which not only consumes materials and energy but also leads to waste of the earlier produced product ${ }^{18,19}$.

Many strategies have been developed to build dynamic mechanisms into soft materials to enable them to reshape $^{20-23}$, self-heal ${ }^{24-31}$, re-assemble ${ }^{32-34}$, be recycled ${ }^{35-39}$, change various properties ${ }^{40,41}$, etc. These material systems are sophisticated, but their transformations are predesigned and are not changeable beyond this design because the materials lack mechanisms to absorb external 'nutrients' to grow and to open new functions. Recently, several types of materials have been developed ${ }^{42-44}$ that can incorporate feeding chemicals via irreversible reactions to increase their size and vary their properties. Such a bioinspired growing strategy for post-modulation can even be applied to different thermosetting materials. In all these cases, however, the change in the size is limited and unidirectional towards materials growth due 
to the irreversible nutrient uptake mechanism, which restricts the flexibility of this strategy in programming material's sizes and properties in both directions of mass accumulation and decomposition.

Compared to irreversible reactions, reversible chemical reactions are also common and their incorporation in the growing materials systems would lead to equilibrium states, in which addition or removal of starting reactants can drive the systems forward to or reverse from the products ${ }^{12}$, showing many features similar to the remarkable self-organization of Planaria. Herein we apply this equilibrium concept to design a Planaria-inspired strategy of enabling materials to grow or degrow to achieve the desired sizes, shapes, compositions, and materials properties simultaneously, in a sustainable and on-demand manner. The idea is demonstrated by silicones made via acid-catalyzed ring-opening polymerization. The resulting polymers retain acidic characteristics and can continuously grow by incorporating supplied substances or degrow through releasing small molecules generated in depolymerization. The composition, shape, and various properties of the materials including mechanical performance, surface morphology, and spectroscopic properties, can be selectively tuned through this reversible growth, suggesting unprecedented opportunity in post-modulation of thermosetting materials.

\section{Strategy and system}

Figure 1B shows our design. The materials consist of crosslinked polymers in a catalyst-dependent equilibration (Figure 1Bi). For growth, polymerizable substances, i.e. monomer and crosslinker, are supplied to the systems by swelling (Figure 1Bii), to drive the polymerization toward the formation of new crosslinked networks. Normally, polymer chains of crosslinked networks will be stretched during the swelling, which hinders further uptake of liquid molecules. In equilibrating polymer networks that are capable of secondary redistribution reactions within the polymer networks, such entropic discomforts will trigger chain exchange between the original and newborn networks, to generate networks with relaxed polymer conformations (Figure 1Biii). As a result of the homogenization, the grown and relaxed polymer network can undergo further growth cycles (Figure 1Biv). Importantly, removing the monomers from the system can drive the polymer networks to shrink (Figure 1Bv), leading to degrowth (Figure 1Bvi). Based on this equilibrium mechanism, the size, composition, and set of properties of the samples can be tuned finely and reversibly, by controlling the availability of polymerizable substances. Besides, the equilibration can be switched off by quenching the catalyst to achieve a stable, desirable product, or turned on again through reactivating the catalyst.

Acid-catalyzed equilibrium of siloxane was selected to demonstrate our growth-degrowth concept. The materials were prepared via acid-catalyzed ring-opening copolymerization of octamethylcyclotetrasiloxane $\left(\mathrm{D}_{4}\right)$ and a star-shaped crosslinker $\left(\operatorname{triD}_{4}\right)$ with trifluoromethanesulfonic acid as catalyst (Figure 1C, Supplementary Scheme 1 and Supplementary Figure 1). The acidic species retained their activity after polymerization and could easily percolate throughout the networks to distribute uniformly and even escape from the sample (Supplementary Figure 2) ${ }^{13-15,45,46}$. To prevent their evaporation and percolation, fillers like carbon black $(\mathrm{CB})$ and silica particles $\left(\mathrm{SiO}_{2}\right)$ that would 
adsorb acidic molecules on their surfaces ${ }^{47}$ (Supplementary Figure 3-5) could be selectively added into the materials. The obtained materials were similar to normal silicone elastomers and could swell upon the uptake of the mixture of their parent monomer and crosslinker (nutrient solution). During swelling, $\mathrm{D}_{4}$ and triD ${ }_{4}$ were homogeneously absorbed (Supplementary Figure 6 and Table 1).

\section{Growth}

To trigger growth, nutrient was supplied to the equilibrium system by immersing the living samples in a nutrient solution. The growth could be divided into three overlapping steps: swelling of the nutrient reactants to increase the size of samples, in-situ polymerization to incorporate these swelled reactants, and chain exchange to release swelling-induced mechanical tension (Figure 1B). Since all processes proceeded readily at room temperature ( $\mathrm{rt}$ ), the samples had already undergone growth during the immersion. Figure 2A shows the weight of the samples during the immersion. For comparison, a deactivated sample, in which the acidic species were neutralized to stop the equilibration (Supplementary section 4.2), was used as a control. The control shows typical swelling kinetics and reaches a stable swelling ratio of 3.45 after $10 \mathrm{~h}$. It completely shrinks back after the absorbed liquid is washed out. In contrast, the weight of the living samples continually increases up to $650 \mathrm{wt} \%$ over the same immersion time, and the net weight after washing is more than doubled compared to the starting weight of the sample and that of the control. This extra increase in weight was attributed to the incorporation of the nutrient reactants into the polymer chains through the growing cycle. The swelled samples were further stored at it in a sealed condition to study the kinetics of the system reaching a new monomer-polymer equilibrium (the polymerization was stopped by hexane washing to remove the nutrient in the sample, which could also be applied to terminate the growth). Growth index, defined as $W_{\text {grown }} / W_{\text {original }}$, where $W_{\text {original }}$ and $W_{\text {grown }}$ are the weights of the starting and grown samples, respectively, was used to describe the kinetics of the equilibration process. Growth index was observed to stabilize after $90 \mathrm{~h}$ (Figure 2B and Supplementary Figure 7), indicating a remarkably slower (nearly an order of magnitude) polymerization step compared to that of swelling. The faster swelling process allowed the sample to integrate nutrients homogeneously. Consequently, the growth was uniform throughout the samples and the original shape of the samples, though scaled in size, was maintained (Figure 2C and Supplementary Figure 8).

To facilitate the understanding of the growth mechanism and quantify the growth kinetics, theoretical analyses were carried out. In this system, the polymer chains were constantly changing due to the ongoing chain propagation and exchange reactions between the original and newborn networks. The dynamic chain model for polymers with transient networks ${ }^{48}$ was thus adopted and expanded to simulate the growth. The model included the following features (see supplementary 4.7 for more details): (i) chain distribution tensor was used to describe the change in the end-to-end distances of the chains due to polymerization and chain-exchange reaction; (ii) the diffusion of monomers and crosslinkers was described by Fick's law; (iii) the concentration of the catalyst was set to be a constant inside the polymer; and (iv) for the reaction kinetics, the polymerization rate was assumed to be proportional to the monomer and catalyst concentrations, and the chain exchange reaction rate was considered to be proportional to 
the chain density. The model was calibrated by fitting the theoretical curve of the swelling kinetics and reaction kinetics to the experimental data (black line in Figure 2A and the solid line in Figure 2B). Then the model was used to predict the growth kinetics of the living polymer with concurrent swelling and reaction, which compares well with the experimental results as shown in the orange line in Figure 2A. The results supported the proposed growth mechanism and made the growth process quantitatively predictable.

The size of the samples could be finely modulated by controlling either immersion time (which correlates with the amount of supplied nutrient) or storage time for a given nutrient uptake (which correlates with the yield of polymerization due to the slower polymerization kinetics of the swollen samples) in a single growth cycle or significantly magnified by repeating the growth process (inset in Figure 2B). Such feature also implied interesting regeneration ability: a cubic piece cut from a cubic parent specimen (P1) could be converted into an exact copy of its parent with the same composition and shape (P2), and this process can be repeated indefinitely with the growing offsprings of either the original parent or its descendants (P3) (Figure 2C).

The composition of the grown samples could be easily tuned by varying the nutrient solution, specifically the concentration of the cross-linker, providing a way to modulate their mechanical properties (Figure 2D). Growth in the same nutrient solution as used for preparing the starting samples led to the same composition and similar mechanical properties, while increasing crosslinker concentration in the nutrient solution produced stiffer grown samples. In contrast to the significant change in Young's modulus, the growth index did not vary noticeably with the change in the cross-linker concentration in the nutrient solution (Supplementary Table 2), suggesting that overall kinetics of the incorporation of the new material from these nutrient solutions remained very similar and fairly independent of the concentration of the crosslinker.

\section{Degrowth}

In the equilibration of siloxane, depolymerization occurs through backbiting reactions to generate small cyclic molecules. ${ }^{15}$ Removal of these depolymerization products from the systems would shift the equilibrium and drive the samples to degrow. Evaporation method was used to remove the volatile small molecules which were collected by a homemade cold trap for ${ }^{1} \mathrm{H}$ NMR and GC-MS analysis (Supplementary Figure 9). Analytical results indicated that these molecules consist of $85.5 \% \mathrm{D}_{4}$ and $14.5 \% D_{5}$ (Supplementary Figure 10, these recycled monomers were pure enough for direct repolymerization to prepare silicone again). Since the starting materials used were $\mathrm{D}_{4}$ and $\operatorname{triD}_{4}$, the formation of other cyclic structures $\left(D_{5}\right)$ supported the proposed mechanism of backbiting depolymerization. Figure 3A shows the evaporation-induced degrowth kinetics of the samples under different conditions with a deactivated sample as a control. While the control keeps $99.8 \%$ of its weight, filler-free and filler-containing samples shrink by $60 \%$ and $85 \%$, respectively, in $180 \mathrm{~h}$. The higher shrinkage in filler-containing systems was attributed to the stabilization by the filler of the otherwise volatile catalyst against evaporation (Supplementary Figure 11). The dynamic chain model presented here for growth was also used to simulate the degrowth process and its kinetics (Supplementary section 
4.10). In this case, the degrowth rate was proportional to the monomer and the catalyst concentrations in the matrix. The theoretical results (solid lines) fit well with the experimental data (Figure 3A).

The degrowth could be deactivated by neutralizing the acid catalyst to generate stable materials and reactivated again by supplying acid catalyst to the deactivated systems (Figure 3B), enabling an "on-off" control. The same control could also be achieved in growth process. It is noteworthy that the removal of nutrient components entrapped in the sample by hexane washing or the storage of the sample in a sealing condition to prevent evaporation of small molecules could be employed to stop the growth and degrowth, respectively, but the sample was still in an "on" active state. Growth or degrowth would occur again when nutrients were provided to the sample or the sample was exposed to air. In contrast, in "off" states, the grown or degrown samples were stable in air as normal silicone elastomers. With degrowth, the samples self-stiffened (Supplementary Figure 12) because the produced crosslinker-like depolymerization products, which were not volatile under the reaction conditions, led to an increase in the degree of crosslinking in the samples.

The degrowth is uniform in the absence of any filler and the depolymerized smaller products maintain their geometric shapes (Figure $3 \mathrm{C}$ ). The process of evaporation-induced degrowth could be divided into two separate steps: i) depolymerization to generate small cyclic molecules and ii) the removal of the generated molecules. Since the latter (22h, Supplementary Figure 13$)$ is significantly faster than the degrowth (180 h, Figure 3A), the degrowth should depend on the former, i.e. depolymerization. For a sample, in which the acidic species triggering depolymerization distributed homogeneously due to the percolation, homogeneous reduction in size is expected, which should lead to a uniform, but smaller, bulk structure of the same shape. To confirm this, we designed a perylenediimide(PDI)-based dye crosslinker (Supplementary Scheme 2) whose nonvolatile and crosslinking nature in networks should fully preclude its redistribution after its integration into the network. When a sample dyed with this crosslinker degrew, it displayed intensive and uniform fluorescence (Figure 3D), implying a homogeneous matrix. After degrowth, the system retained its acidic character and could integrate nutrient solution to grow back to its original state (Figure 3C, D).

Degrowth was driven by the evaporation of small cyclic products occurring from the surface and inevitably generating a concentration gradient of the active species. In the filler-free systems, such gradient was significantly reduced due to the percolation of the active species (Figure 3E). Since fillers like $\mathrm{CB}$ or $\mathrm{SiO}_{2}$ could immobilize the acidic species, we expected that the gradient could be preserved in the presence of fillers, finally resulting in a heterogeneous degrowth process (Figure 3E). To prove this hypothesis, we investigated the bulk structure of degrown dyed $\mathrm{SiO}_{2}$ /living siloxane composites with a filler-free sample as a control (Figure 3F). The control shows uniform matrices in the as-prepared, degrown and regrown states due to the homogenization of percolating species in the sample. In the $\mathrm{SiO}_{2}$ /siloxane composites, the dye-functionalized crosslinker would be readily adsorbed on the acidic $\mathrm{SiO}_{2}$ through the imide(PDI)-acid interaction to make the $\mathrm{SiO}_{2}$ particles brightly fluorescent (Figure $3 \mathrm{Fii}$ and Supplementary Figure 14-16). These bright particles distribute homogeneously in the as-prepared sample but form a gradient in the degrown sample, i.e. more fillers in the outer region, suggesting faster 
degrowth in the exterior. To further confirm the mechanism, composites made from hydrophobic polytetrafluoroethylene particles that could not immobilize acidic species were subjected to the same treatment. In these composites, matrices rather than particles are brightly fluorescent, which remain homogenous throughout different states (Supplementary Figure 16). Interestingly, a homogeneous structure formed again when the degrown $\mathrm{SiO}_{2}$ / siloxane composite was regrown, implying a memory effect in the heterogeneous degrowth-growth cycle (Figure 3Fii). We attributed the memory effect to the relative immobility of the particles in the matrices due to their big sizes. These fixed particles allowed the regions showing faster depolymerization in degrowth to undergo similar faster polymerization in growth (Figure 3E). To confirm this mechanism, we compared the growth rates of the degrown samples with different shrinking ratios $(10 \%, 15 \%$, and $20 \%)$. The degrown sample with a higher shrinking ratio (higher concentration of acid-adsorbing $\mathrm{SiO}_{2}$ ) shows faster growth when they were grown in the same nutrient solution (Supplementary Figure 17).

\section{Controlling the material properties of grown-degrown samples}

Several experiments were conducted to demonstrate the potential of the growing-degrowing strategy in post-manipulation of material's size, shape, and various material properties (Figure 4 and Supplementary Section 4.17-4.21). 1) By taking advantage of homogeneous growth, a series of scaled replicas of surface micro-patterns with programmable sizes could be prepared from a single substrate (Figure 4A). When the microstructures were magnified, their aspect ratio was well maintained. 2) In contrast to the uniform growth, the growth of the samples also showed incredible adaptability to geometric restriction. As shown in Figure 4B, two small dyed pieces placed in two corners of an $\mathrm{H}$-shape chamber, respectively, would grow along the wall when a dye-free nutrient solution was supplied. During the growth, the samples indeed continually reshaped themselves to adapt to the mold and finally joined together, resulting in an integrated $\mathrm{H}$-shape elastomer. The integrated product would show uniform fluorescence throughout the sample under irradiation, indicating that the dye crosslinkers in the pieces have been homogeneously redistributed during the growth. 3) Importantly, the final seamless merging of the two separately growing parts into a uniform $\mathrm{H}$-shape also suggested an effective self-healing ability. Indeed, further tests indicated that all the as-prepared, degrown, and regrown samples display excellent selfhealing ability at it without any external stimulus due to the ongoing rearrangements of siloxane networks (Figure 4C). All the samples could self-heal from the pieces that had been completely cleaved and the healed samples exhibit nearly the same stress-strain curves as the intact samples. 4) The heterogenous degrowth-growth could be utilized to switch surface morphologies. For example, when a $\mathrm{SiO}_{2}$ /siloxane film with a translucent and smooth surface was allowed to degrow to expose the undegradable fillers, an opaque rough coating was obtained (Supplementary Figure 18). Since the fillers were still coated with siloxane (Supplementary Figure 18), the sample switches from hydrophobic to superhydrophobic (Figure 4D, top row). 5) Moreover, the superhydrophobicity could be patterned on the surface by using masks to control localized molecular evaporation resulting in the creation of regionally degrown superhydrophobic patches (see Schematic in Fig 4D and Supplementary Figure 19). The patterns could be erased by growth and rewritten again. Stable patterns could be obtained by 
deactivation treatment (Supplementary Figure 20). 6) The shrinkage in degrowth can also be harnessed to create actuated materials (Supplementary Figure 21), which was demonstrated in controllable deformation of the degrown and regrown samples (Figure 4E). Such deformation was also reversible in the heterogenous degrowth-growth cycle.

\section{Conclusion}

Inspired by the reversible growth of Planaria, we have developed a fundamentally new strategy of enabling thermoset materials to continuously modulate their sizes, shapes, compositions, and set of properties on demand. The strategy is based on the expression of monomer-polymer equilibration from molecular to macroscopic material levels. By supplying or removing polymerizable substances to shift the equilibria, the materials were induced to grow or degrow. The growth or degrowth can be reversibly turned off to yield stable materials. By the availability of fillers, the degrowing-growing processes can be selected to be uniform or heterogeneous. Taking full advantage of these mechanisms, we have demonstrated many appealing features of the resulting materials including regeneration ability, environment adaptivity, self-healing ability, and switchability in surface morphologies, optical properties, and shapes. The acid-catalyzed siloxane polymerization/depolymerization demonstrated here is only one representative example of many reversible processes that can occur in polymer materials, even though it is a widely used material for various applications, which suggests many immediate practically important opportunities. We emphasize the applicability of our strategy to other polymer systems showing monomer-polymer equilibration, such as polyester, polyolefin, polysulfide, polyether, polyamide, etc ${ }^{12,16,17}$. Besides post-modulating materials for more sustainable use, reversible growth also represents a new attractive method for material fabrication in which waste generation is minimized. We envision this new paradigm to have significant potential in smart materials for meeting many emerging challenges, like the reuse of thermoset materials.

\section{Declarations}

\section{Data Availability}

The data that support the findings of this study are available from the corresponding author on request.

Acknowledgments: The work was originated and supported by the US Department of Energy (DOE), Office of Science, Basic Energy Sciences (BES) under award number DE-SC0005247, and was further financially supported by the National Natural Science Foundation of China $(51973023,52073175$, 52003035), Sichuan Science and Technology Program (2021JDRC0014 and 2021JDRC0106), and Shanghai Pujiang Program (20PJ1411200).

Author contributions: J.C., J.A., and Y.Z. conceived the concept. J.C. and J.A. supervised the project. X.Z., Y.Z., J.C., L.Y., Y.C., and B.P. conducted the experiments. H.Z. and Y.H. conducted the simulation. M.A. 
analyzed data. J.C., X.Z., Y.Z., H.Z., Y.H., M.A, and J.A. wrote the manuscript. All authors contributed to the analysis and discussion of the data.

Competing Interests: The authors declare no competing interests.

\section{Additional information}

Supplementary information is available for this paper.

Correspondence and requests for materials should be addressed to J.C. and J.A.

Peer review information

Reprints and permissions information is available at www.nature.com/reprints.

\section{References}

1 Ivankovic, M. et al. Model systems for regeneration: planarians. Development 146, dev167684 (2019).

2 Baguñà, J. \& Romero, R. Quantitative analysis of cell types during growth, degrowth and regeneration in the planarians Dugesia mediterranea and Dugesia tigrina. Hydrobiologia 84, 181-194 (1981).

3 Zheng, N., Xu, Y., Zhao, Q. \& Xie, T. Dynamic Covalent Polymer Networks: A Molecular Platform for Designing Functions beyond Chemical Recycling and Self-Healing. Chem. Rev. 121, 1716-1745 (2021).

4 Lutz, J.-F., Lehn, J.-M., Meijer, E. W. \& Matyjaszewski, K. From precision polymers to complex materials and systems. Nat. Rev. Mater. 1, 1-14 (2016).

5 Yang, Y. \& Urban, M. W. Self-healing polymeric materials. Chem. Soc. Rev. 42, 7446-7467 (2013).

6 Scheutz, G. M., Lessard, J. J., Sims, M. B. \& Sumerlin, B. S. Adaptable crosslinks in polymeric materials: resolving the intersection of thermoplastics and thermosets. J. Am. Chem. Soc. 141, 1618116196 (2019).

7 Zhang, Z. P., Rong, M. Z. \& Zhang, M. Q. Polymer engineering based on reversible covalent chemistry: A promising innovative pathway towards new materials and new functionalities. Prog. Polym. Sci. 80, 39-93 (2018).

8 Maeda, T., Otsuka, H. \& Takahara, A. Dynamic covalent polymers: reorganizable polymers with dynamic covalent bonds. Prog. Polym. Sci. 34, 581-604 (2009). 
9 Fortman, D. J. et al. Approaches to sustainable and continually recyclable cross-linked polymers. ACS Sustain. Chem. Eng. 6, 11145-11159 (2018).

10 Lendlein, A. \& Kelch, S. Shape-memory polymers. Angew. Chem. Int. Ed. 41, 2034-2057 (2002).

11 Moulin, E., Faour, L., Carmona-Vargas, C. C. \& Giuseppone, N. From Molecular Machines to Stimuli-Responsive Materials. Adv. Mater. 32, 1906036 (2020).

12 Elias, H.-G. in Polymerization Equilibria in Macromolecules: Volume 2: Synthesis, Materials, and Technology 557-581 (Springer US, 1984).

13 Kantor, S. W., Grubb, W. T. \& Osthoff, R. C. The mechanism of the acid-and base-catalyzed equilibration of siloxanes. J. Am. Chem. Soc. 76, 5190-5197 (1954).

14 Hurd, D. T. On the mechanism of the acid-catalyzed rearrangement of siloxane linkages in organopolysiloxanes. J. Am. Chem. Soc. 77, 2998-3001 (1955).

15 Chang, P. S. \& Buese, M. A. Silicone networks prepared via a living percolation mechanism: postgelation structure for networks with a variety of junction functionalities. J. Am. Chem. Soc. 115, 11475-11484 (1993).

16 Monfette, S. \& Fogg, D. E. Equilibrium ring-closing metathesis. Chem. Rev. 109, 3783-3816 (2009).

17 Greer, S. C. Physical chemistry of equilibrium polymerization. J. Phys. Chem. B 102, 5413-5422 (1998).

18 Rahimi, A. \& García, J. M. Chemical recycling of waste plastics for new materials production. Nat. Rev. Chem. 1, 1-11 (2017).

19 Walther, A. From responsive to adaptive and interactive materials and materials systems: A roadmap. Adv. Mater. 32, 1905111 (2020).

20 Lendlein, A., Jiang, H., Jünger, O. \& Langer, R. Light-induced shape-memory polymers. Nature 434, 879-882 (2005).

21 Xie, T. Tunable polymer multi-shape memory effect. Nature 464, 267-270 (2010).

22 Pei, Z. et al. Mouldable liquid-crystalline elastomer actuators with exchangeable covalent bonds. Nat. Mater. 13, 36-41 (2014).

23 Scott, T. F., Schneider, A. D., Cook, W. D. \& Bowman, C. N. Photoinduced plasticity in cross-linked polymers. Science 308, 1615-1617 (2005).

24 White, S. R. et al. Autonomic healing of polymer composites. Nature 409, 794-797 (2001). 
25 Chen, X. et al. A thermally re-mendable cross-linked polymeric material. Science 295, 1698-1702 (2002).

26 Cordier, P., Tournilhac, F., Soulié-Ziakovic, C. \& Leibler, L. Self-healing and thermoreversible rubber from supramolecular assembly. Nature 451, 977-980 (2008).

27 Burnworth, M. et al. Optically healable supramolecular polymers. Nature 472, 334-337 (2011).

28 Montarnal, D., Capelot, M., Tournilhac, F. \& Leibler, L. Silica-like malleable materials from permanent organic networks. Science 334, 965-968 (2011).

29 Li, C.-H. et al. A highly stretchable autonomous self-healing elastomer. Nat. Chem. 8, 618-624 (2016).

30 Chen, Y., Kushner, A. M., Williams, G. A. \& Guan, Z. Multiphase design of autonomic self-healing thermoplastic elastomers. Nat. Chem. 4, 467 (2012).

31 Billiet, S. et al. Triazolinediones enable ultrafast and reversible click chemistry for the design of dynamic polymer systems. Nat. Chem. 6, 815-821 (2014).

32 Korevaar, P. A. et al. Pathway complexity in supramolecular polymerization. Nature 481, 492-496 (2012).

33 Boekhoven, J., Hendriksen, W. E., Koper, G. J. M., Eelkema, R. \& van Esch, J. H. Transient assembly of active materials fueled by a chemical reaction. Science $349,1075-1079$ (2015).

34 Qian, X. et al. Artificial phototropism for omnidirectional tracking and harvesting of light. Nat. Nanotechnol. 14, 1048-1055 (2019).

35 García, J. M. et al. Recyclable, strong thermosets and organogels via paraformaldehyde condensation with diamines. Science 344, 732-735 (2014).

36 Hong, M. \& Chen, E. Y.-X. Completely recyclable biopolymers with linear and cyclic topologies via ring-opening polymerization of $\mathrm{y}$-butyrolactone. Nat. Chem. 8, 42 (2016).

37 Yuan, Y. et al. Multiply fully recyclable carbon fibre reinforced heat-resistant covalent thermosetting advanced composites. Nat. Commun. 8, 1-11 (2017).

38 Christensen, P. R., Scheuermann, A. M., Loeffler, K. E. \& Helms, B. A. Closed-loop recycling of plastics enabled by dynamic covalent diketoenamine bonds. Nat. Chem. 11, 442-448 (2019).

39 Häußler, M., Eck, M., Rothauer, D. \& Mecking, S. Closed-loop recycling of polyethylene-like materials. Nature 590, 423-427 (2021). 
40 Wojtecki, R. J., Meador, M. A. \& Rowan, S. J. Using the dynamic bond to access macroscopically responsive structurally dynamic polymers. Nat. Mater. 10, 14-27 (2011).

41 Aida, T., Meijer, E. \& Stupp, S. Functional supramolecular polymers. Science 335, 813-817 (2012).

42 Matsuda, T., Kawakami, R., Namba, R., Nakajima, T. \& Gong, J. P. Mechanoresponsive selfgrowing hydrogels inspired by muscle training. Science 363, 504-508 (2019).

43 Chen, M. et al. Living additive manufacturing: transformation of parent gels into diversely functionalized daughter gels made possible by visible light photoredox catalysis. ACS Cent. SCi. 3, 124134 (2017).

$44 \quad$ Xue, L. et al. Light-regulated growth from dynamic swollen substrates for making rough surfaces. Nat. Commun. 11, 1-9 (2020).

45 Pouget, E. et al. Well-architectured poly (dimethylsiloxane)-containing copolymers obtained by radical chemistry. Chem. Rev. 110, 1233-1277 (2010).

46 Zheng, P. \& McCarthy, T. J. A Surprise from 1954: Siloxane Equilibration Is a Simple, Robust, and Obvious Polymer Self-Healing Mechanism. J. Am. Chem. Soc. 134, 2024-2027 (2012).

47 Zhou, X., Zhang, X., Zhao, H., Krishnan, B. P. \& Cui, J. Self-Healable and Recyclable Tactile Force Sensors with Post-Tunable Sensitivity. Adv. Funct. Mater. 30, 2003533 (2020).

48 Vernerey, F. J., Long, R. \& Brighenti, R. A statistically-based continuum theory for polymers with transient networks. J. Mech. Phys. Solids 107, 1-20 (2017).

\section{Figures}




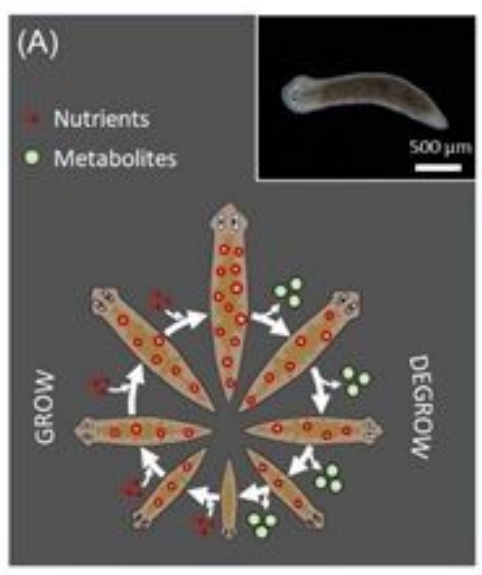

(C)

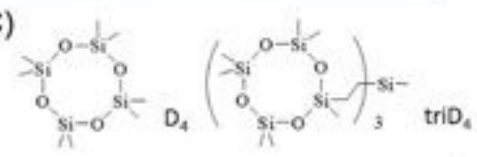

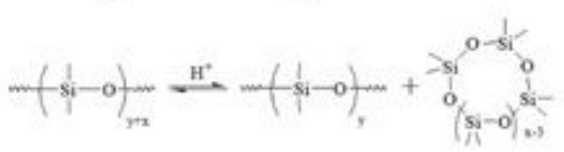

(B)

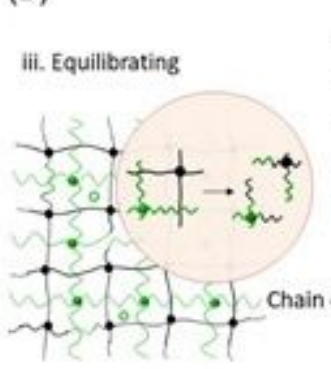

Polymerization

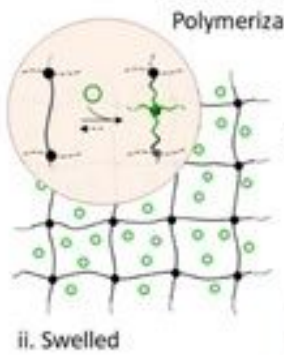

ii. Swelled iv. Grown

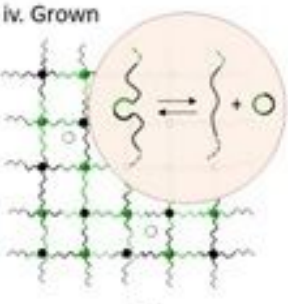

Monomer/crosslinker

v. Shrinking

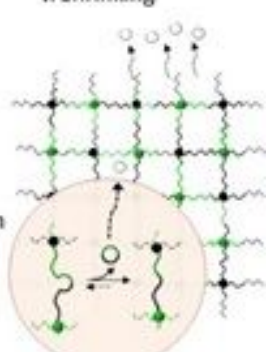

Depolymerization

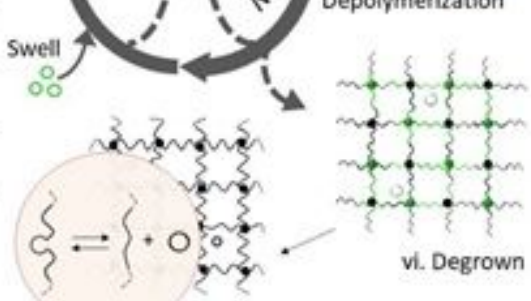

i. Starting

\section{Figure 1}

Planaria-inspired strategy of enabling crosslinked polymers to grow and degrow. A) The growth and degrowth of the Planarians. Inset displays a living Planaria. B) Design of the growing and degrowing molecular mechanism of crosslinked polymeric materials. Growing process: a starting sample (i) swelled the mixture of monomer and crosslinker to get the swelled sample (ii); polymerization and chain exchange reactions occurred in the equilibrating sample (iii) to generate the grown sample (iv). Degrowing process: removal of the monomer by evaporation induced the sample to shrink (v) and then generate the degrown product (vi). C) D4 monomer and triD4 crosslinker used for making living siloxane elastomer and demonstrating the acid-catalyzed monomer-polymer equilibration. 
A

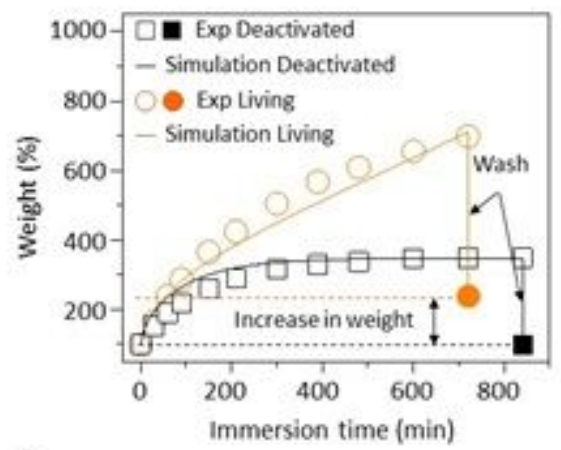

C

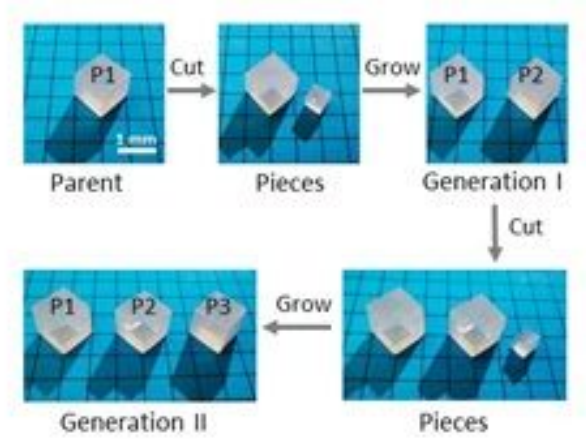

B

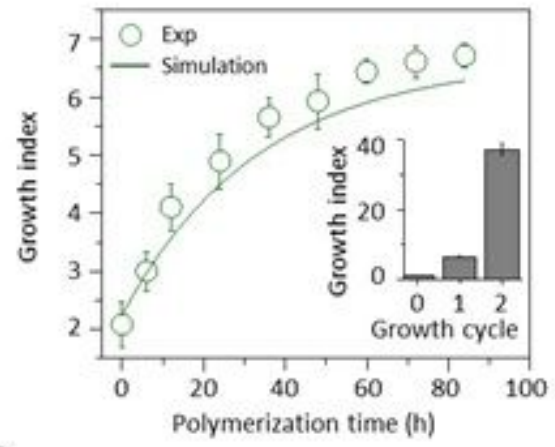

D

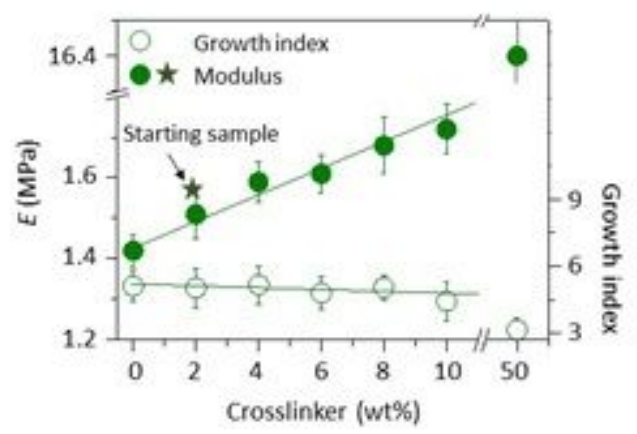

Figure 2

Growth of the living siloxane elastomers. A) Experimental and theoretical time-dependent weight of living and deactivated samples immersed in the nutrient solution. Hexane was used to wash out the nutrient solution. The solid square and circle labels show the weights after washing. B) Experimental and theoretical growth index of grown products under different conditions. The samples were immersed in the nutrient solution for $10 \mathrm{~h}$ and then stored in sealed bottles to allow the polymerization and homogenization to progress for different times, followed by hexane washing. A full growth cycle included $10 \mathrm{~h}$ immersion and $90 \mathrm{~h}$ storage. Growth index: Wgrown/Woriginal. C) Regeneration of the living samples. A cubic sample (1st generation parent, P1) was cut to yield a smaller cubic piece which was grown into a sample (P2) with the same size and shape as P1. The P2 was then used as the 2nd generation parent to regenerate the generation II sample (P3). D) Young's modulus and growth index of grown products obtained from nutrient solutions with different crosslinker concentrations. The nutrients in A), B), and C) contained $2 \mathrm{wt} \%$ triD4. The data in B) and D) were obtained from eight independent measurements. Error bars are standard error of the mean (s.e.m.). 
A

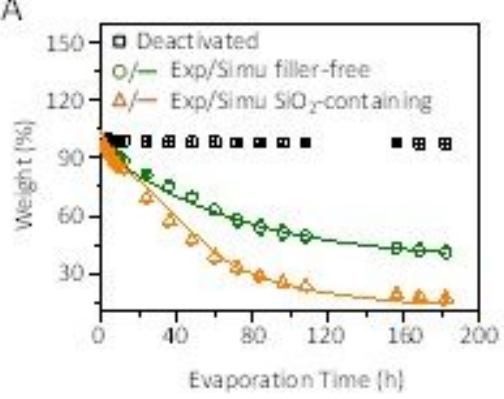

B

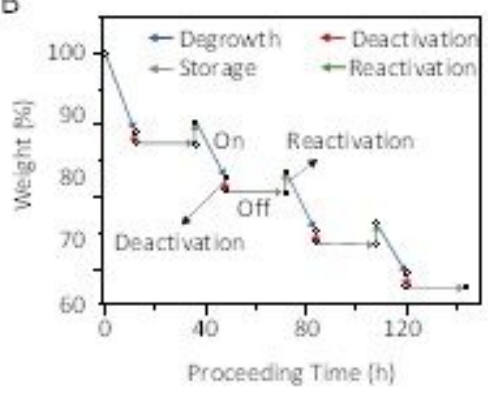

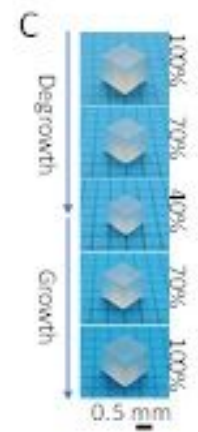

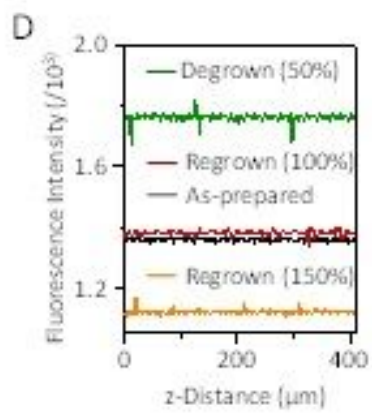

E

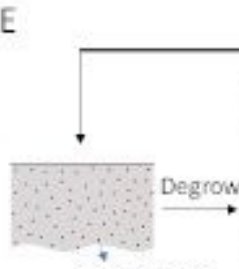

percolatable

- Active species

- Cyclic small molecule

- Nano-filler

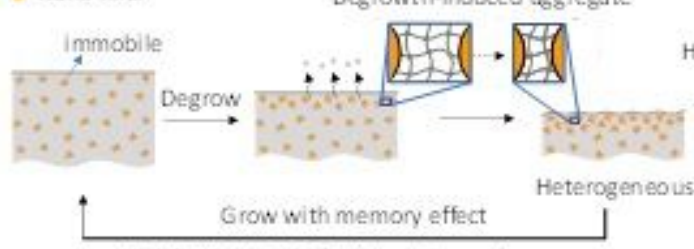

High [active species] $\rightarrow$ Fast degrow/grow
Grow

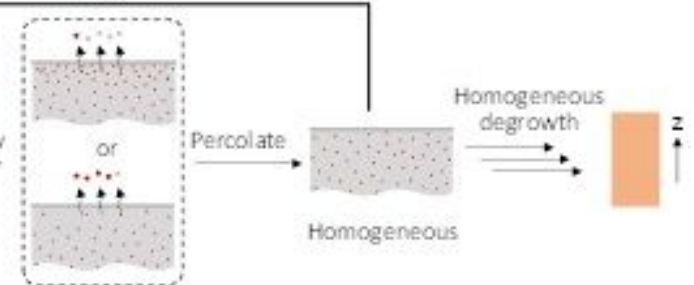

egrowth-induced aggregate

Heterogeneous degrowth

\section{Figure 3}

Degrowth of the living siloxane elastomers. A) Time-dependent weight of different samples in air at rt. Deactivated: deactivated sample; Exp: experimental; Simu: simulated; filler-free: living siloxane elastomer; SiO2-containing: living SiO2/siloxane composite. The experimental data were obtained from eight independent measurements. Error bars are s.e.m. B) The weight change of a siloxane elastomer in multiple activation-deactivation cycles. The living sample was deactivated by washing the acidic species with triethylamine and re-activated by adding trifluoromethanesulfonic acid. C) A cubic sample in a degrowth-growth cycle. D) Fluorescence intensities of a dyed living siloxane elastomer at different states. The as-prepared sample was first degrown to $50 \%$ of its initial weight, followed by being regrown to $100 \%$ and then to $150 \%$ of its initial weight. E) Schematic mechanisms of homogeneous and heterogeneous degrowth-growth. F) Vertical cross-section fluorescence images of a dyed living siloxane elastomer (i) and a dyed $\mathrm{SiO} 2 /$ siloxane composite (ii) at different states. 
A

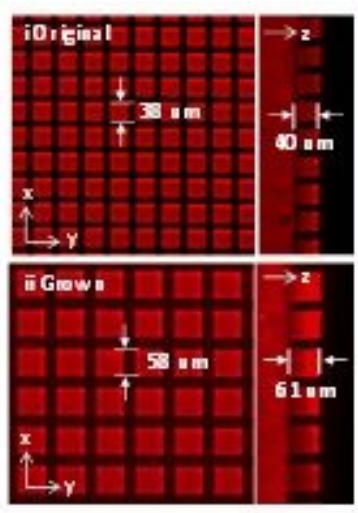

C

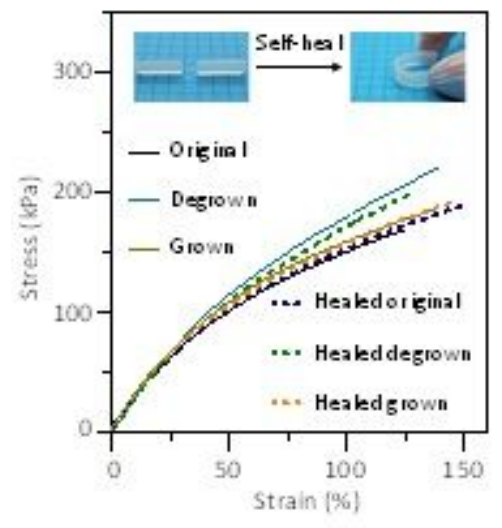

B

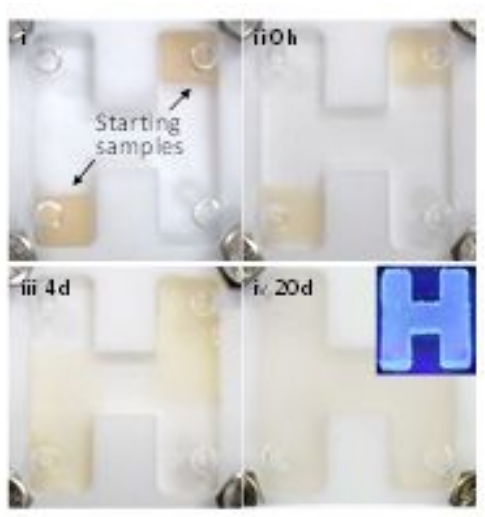

D

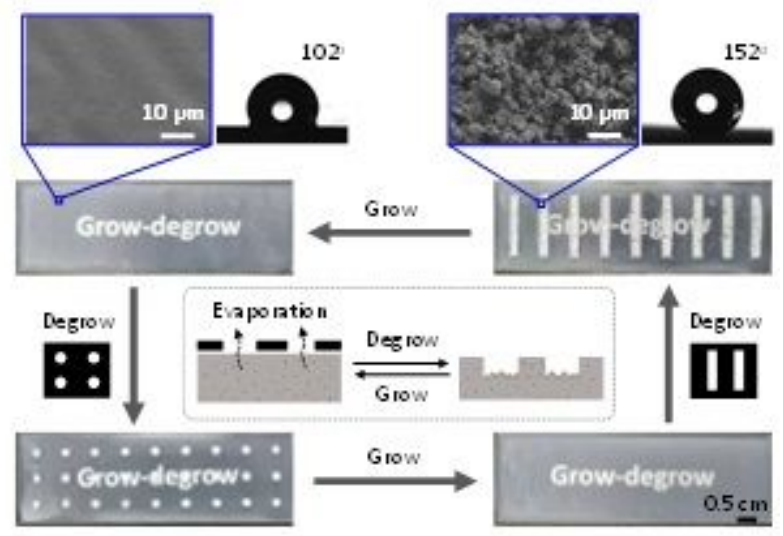

E
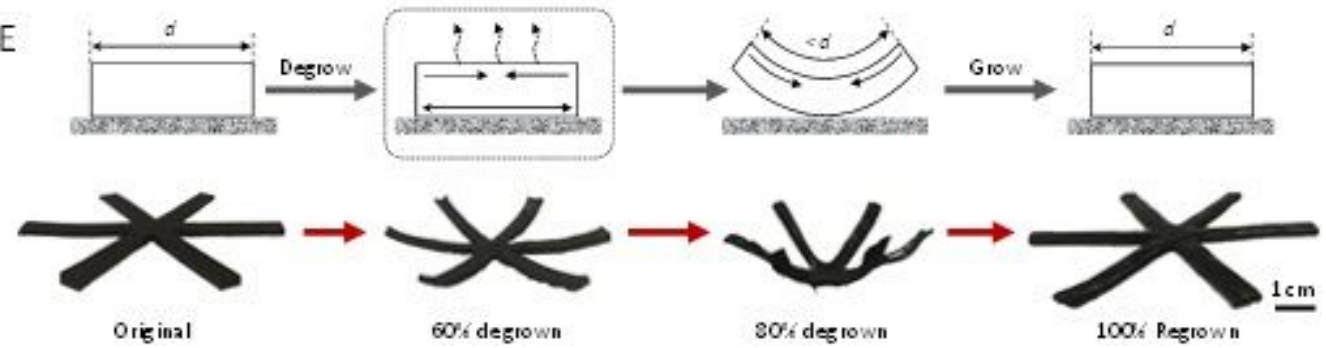

Figure 4

Structure modulation by growth and degrowth. A) Growth of dyed microposts made from living siloxane elastomer: fluorescence image (Left: horizontal cross-section; Right: vertical cross-section) of the original sample (i) and its grown product (ii); (iii) Plot of the size of the microposts against the cubic root of the growth index. B) Confinement growth of two dyed samples (i) in a chamber for different times (ii-iv): h: hour; d: day. Dye-free nutrient was used. Inset: the final product showed homogeneous emission under UV irradiation. C) Demonstration of self-healing and the typical stress-strain curves of different state samples. Original: as-prepared sample; Degrown: degrown sample (70\% of the initial weight); Grown: grown sample (with a grown index of 1.3). The healed samples were obtained by putting two pieces of the samples together and stored overnight in a sealed condition. Typical uniaxial tensile tests with a loading rate of $20 \mathrm{~mm} / \mathrm{min}$ were conducted to get the stress-strain curves. D) Reversible patterning of a SiO2/siloxane film. The surface was observed by SEM and the water contact angles were obtained from a big enough non-patterned sample. E) Deformation of a CB/siloxane sample in degrowth-growth. 


\section{Supplementary Files}

This is a list of supplementary files associated with this preprint. Click to download.

- Supplementaryinformation.docx 